\title{
Coping style, psychological distress, risk perception, and satisfaction in subjects attending genetic counselling for hereditary cancer
}

\author{
K Nordin, A Lidén, M Hansson, R Rosenquist, G Berglund
}

co has been known for some time that cancer is more common in some families than in others. ${ }^{1}$ The rapid development of human genetics research has shown two highly penetrant breast and ovarian cancer susceptibility genes, $B R C A 1$ and BRCA2. ${ }^{23}$ Approximately $5 \%$ of all breast cancers are the result of inherited genetic mutations of these genes. ${ }^{4}$ Among colorectal cancers, between 2 and $10 \%$ are the result of known genetic mutations. ${ }^{5}$ The most common genetic mutations are located in the $A P C$ gene, which causes familial adenomatous polyposis (FAP) and in the DNA mismatch repair genes, predisposing to hereditary non-polyposis colorectal cancer (HNPCC). However, the genetic mutations are still unknown in a large number of families with hereditary breast and colorectal cancers.

As a result of the recent developments in DNA testing and the knowledge that cancer can be hereditary, it is now possible to offer genetic counselling to people within identified "cancer families", and to inform them about their assumed increased risk for developing cancer. One of the most important components in genetic counselling is the provision of information. Extensive research was performed early on principles for providing patient information in a general medical setting. ${ }^{6}$ This type of information is usually fairly simple and concerns mainly the patient him/herself. In contrast, the information in genetic counselling is often highly complex, including risk estimates and aspects of heredity involving family members. The recall of risk estimates after counselling among women with a family history of cancer has been found to be poor. ${ }^{78}$ Studies have been performed of the psychological impact of informing subjects about their risk for developing hereditary cancer, ${ }^{9}$ showing that their psychological reactions are not related directly to the risk estimates. ${ }^{10}{ }^{11}$ In subjects who were DNA tested for Huntington's disease, psychological reactions were predicted by their psychological status before the test rather than by the test result itself. ${ }^{12}{ }^{13}$ One explanation for such discrepancies may be sought in the field of coping with stressful situations. Theories of coping postulate that a person's reaction to a stressful situation is moderated by his/her ability to handle the threat and the resulting reactions.${ }^{14}$ The relevance of coping for understanding psychological well being among cancer patients has been shown in several studies. ${ }^{15-17}$

An important aspect of the ability to cope with a potentially serious illness is the desire for information about its various facets. ${ }^{18}$ Some people, referred to as Monitors, have a tendency to search out information and focus on health threats, whereas others (Blunters) have a tendency to avoid information. ${ }^{1920}$ It has been proposed that in stressful situations, it is important to adjust the information given to the person's coping style. The provision of a lot of information reduces psychological distress in Monitors while less information does so in Blunters. ${ }^{21}{ }^{22}$ Monitoring and blunting have been studied in a number of health care situations and found to be of clinical relevance. ${ }^{23}$ Phipps and Zinn ${ }^{18}$ investigated the relation between coping style and mood state in a group of women undergoing amniocentesis. Monitors experienced more distress than Blunters, both before and during the procedure, but their distress decreased after communication of the test result. The period of waiting for the result of the amniocentesis was found to be stressful for Monitors when no information about the test result was available. In women with an increased risk for breast/ovarian cancers, a high monitoring score was associated with greater psychological distress while waiting for the test results. ${ }^{24}$ Schwartz et al ${ }^{25}$ found that high monitoring scores were associated with high perceived risk for ovarian cancer in a group of women with at least one first degree relative with the disease. Most studies of Monitors and Blunters have used a general measure, the Miller Behavioural Style Scale (MBSS). ${ }^{19}$ A growing interest in measuring patients' coping styles, especially in medical settings, has resulted in the construction of an instrument for assessing these coping styles for the domain of medical threat only, the Threatening Medical Situations Inventory (TMSI). ${ }^{26} 27$ Another reason for developing an alternative method is that medical patients may find themselves in despair and often get irritated over the perceived irrelevant, hypothetical scenarios of the MBSS (for example, being taken hostage by a group of terrorists). ${ }^{27}$

Patient satisfaction with communication in medical settings is not a simple function of the communication skills of the staff. ${ }^{28}$ Rather, the patient's style of coping in terms of seeking out or avoiding information is a moderator of the outcome of patient-staff communication. Patients are not only less distressed but also more satisfied with the information if it is adapted to their coping style. ${ }^{29}{ }^{30}$ Thus, it is important to find out the extent to which monitoring and blunting tendencies play a role in genetic counselling, especially since complex information is an essential part in the counselling of "cancer families".

The aim of the present study was to investigate the relationship between, on the one hand, coping styles (monitoring/blunting) among subjects attending genetic counselling and, on the other, psychological distress, risk perception, and satisfaction with counselling. The following specific questions were raised. What is the relationship between two measures of coping styles, one of which represents a measure of general monitoring/blunting tendencies and the other a measure developed specifically for threatening medical situations? Are there any differences between Monitors and Blunters with respect to (1) psychological distress before,

Abbreviations: FAP, familial adenomatous polyposis; HNPCC, hereditary non-polyposis colorectal cancer; MBSS, Miller Behavioural Style Scale; TMSI, Threatening Medical Situations Inventory; HADS, Hospital Anxiety and Depression Scale; SCS, Satisfaction with Genetic Counselling Scale; $M B$, monitoring minus blunting 
directly after, and six months after a session of genetic counselling, (2) their cancer risk assessments before and after the counselling, and (3) their satisfaction with counselling? Is there any relationship between satisfaction with counselling and psychological distress before, directly after, and six months after the counselling?

\section{PATIENTS AND METHODS \\ Patients}

A consecutive series of subjects referred for genetic counselling for breast, ovarian, or colorectal cancer at the oncogenetic outpatient clinic at Uppsala University Hospital were asked to participate. Recruitment started in September 1999 and ended in June 2001. Out of 67 eligible subjects, 63 (94\%) participated. Of the four who did not participate, two considered the questionnaires too difficult to complete, one had recently gone through surgery, and one did not want to participate for personal reasons. The mean age of the participants was 43.5 years (range 19-73) and 58 were female and five male. Forty-five subjects $(71 \%)$ were counselled for suspected hereditary breast cancer (43 females, two males), five $(8 \%)$ for ovarian cancer, and $13(21 \%)$ for colorectal cancer ( 10 females, three males).

\section{Measures \\ Coping \\ The Miller Behavioural Style Scale (MBSS)}

The MBSS describes four threatening scenarios and the respondent is instructed to imagine her/himself in these: at the dentist, taken hostage by a group of armed terrorists, the threat of job loss, and flying in an aeroplane when something goes wrong. ${ }^{19}$ An abbreviated version of the MBSS with only two scenarios (dentist and job loss) has been developed and validated $^{31}$ and was used in the present study. Participants were asked to select all response alternatives that apply to them among eight behaviours related to each situation. Four items are related to monitoring and four to blunting, forming two subscales. Summing all items endorsed on the monitoring subscale yields the monitoring score, and the corresponding computation gives the blunting score. The sum score, derived by subtracting the total blunting score from the total monitoring score, was used for categorisation of the participants. Thus, they were categorised as Monitors or not, on the basis of whether they scored above or below the median of the sum score. ${ }^{32}$

\section{The Threatening Medical Situation Inventory (TMSI)}

The TMSI includes four descriptions of threatening medical situations: vague, suspicious headache; being diagnosed as hypertensive; choosing uncertain heart surgery; and a sudden appendicitis operation. ${ }^{27}$ In order to ensure that the whole range of potential medical stressors was covered, these situations diverge with respect to two important stress parameters: controllability and predictability. Being diagnosed with hypertension is both, a sudden appendicitis operation is predictable but not controllable, choosing an uncertain heart operation is controllable but not predictable, and vague, suspicious headache is neither predictable nor controllable. Each TMSI situation is followed by three blunting and three monitoring items, arranged in random order, to be answered on a five point Likert scale $(\mathrm{l}=$ not at all applicable to me, $5=$ strongly applicable to me). Total monitoring and blunting scores were calculated by adding up the scores of the relevant items (range for both subscales 12-60). Patients were categorised as Monitors or Blunters on the basis of the sum score, using the same procedure as described for the MBSS.

\section{Psychological distress}

The Hospital Anxiety and Depression Scale (HADS) was used for assessment of anxiety and depression. It consists of two subscales: depression (seven items) and anxiety (seven items). Subscale scores range from 0 (no distress) to 21 (maximum distress). ${ }^{33}$ Physical signs of anxiety and depression are not included. The HAD scale has been found to be valid for use with somatic patients. ${ }^{34} 35$

A numerical 1-7 scale with the end points defined as "no worry at all" and "worst possible worry" was used to assess patient worry immediately before and after the counselling.

\section{Risk assessment}

Participants completed a risk assessment form based on the work by Evans et al. ${ }^{76}$ Individual risk perceived by the person in counselling was expressed both in gambling odds ratio (for example, $1 / 2,1 / 3,1 / 50,1 / 100)$ and percentage $(50 \%, 35 \%, 2 \%$, $1 \%)$. The physician completed a corresponding form concerning the individual risk estimate communicated. The risk perceived by the subject was compared to the risk estimated by the physician. In the present study, to be categorised as "correct", a participant's estimate was allowed to deviate one step (above or below) the risk figure given by the physician, except for the three highest risk figures, $35 \%, 50 \%$, and $80 \%$, for which exact correspondence was required.

\section{Satisfaction}

The Satisfaction with Genetic Counselling Scale (SCS) (short form) consists of 12 items measuring three dimensions of satisfaction, "Instrumental" (for example, "Did the doctor explain your condition to you clearly?") (three items), "Affective" (for example, "Did the doctor listen to what you had to say?") (three items), and "Procedural" (for example, "How did you rate the length of time you waited since you first contacted the clinic until your visit?") (three items). ${ }^{37}$ The ranges for all three dimensions are 3-12. Three individual items (range 1-4) measuring satisfaction with the information ("Information"), met expectations ("Expectation"), and overall satisfaction with the counselling ("Overall satisfaction") will be presented separately.

\section{Procedure}

Subjects who agreed to participate completed questionnaires one week before (coping questionnaires and HADS), in the waiting room immediately before (numerical 1-7 worry scale, risk assessment), after the counselling (numerical 1-7 worry, risk assessment), and after six months (HADS). The Satisfaction with Counselling Scale (SCS) was to be completed at home within a few days after the visit and then returned by mail. Immediately after the participant left the counselling room, the physician filled out the risk assessment form. The physician was asked to rate each participant's life time risk for developing the cancer screened for, that is, the risk estimate that the physician had communicated to the patient in counselling.

\section{Statistical analysis}

Pearson product moment correlations (r) and paired and unpaired $t$ tests were used. Agreement between the categorisations of participants as Monitors or not with the MBSS and the TMSI was calculated using Cohen's Kappa statistics ( $\kappa)$. A one way Anova was performed to compare subjects who overestimated, underestimated, or correctly estimated their risk for developing cancer. Differences between groups (Monitors/ non-Monitors) over assessments (time) were subjected to analysis of variance using Anova. Post hoc testing between and within groups was performed by the Fischer least significant difference (LSD) test.

\section{RESULTS}

Relationship between two measures of coping styles, the MBSS and the TMSI

The correlation between the MBSS and the TMSI monitoring scores was $\mathrm{r}=0.45(\mathrm{p}<0.01)$ and the corresponding correlation for blunting was $r=0.06$ (NS). The number of patients 
Table 1 Agreement between categorisation of Monitors and non-Monitors using the TMSI and the MBSS

\begin{tabular}{llll}
\hline & \multicolumn{2}{l}{ TMSI Monitors } & \\
\cline { 2 - 3 } & Yes & No & Total \\
\hline MBSS Monitors & $18(34 \%)$ & $13(25 \%)$ & $31(58 \%)$ \\
Yes & $9(17 \%)$ & $13(24 \%)$ & $22(42 \%)$ \\
No & $27(51 \%)$ & $26(49 \%)$ & $53(100 \%)$ \\
Total & & &
\end{tabular}

Cohen's Kappa $=0.17$

categorised as "Monitors" was 58\% using the MBSS and 51\% using the TMSI. However, the agreement between the MBSS and the TSMI categorisations was low ( $\kappa 0.17$ ) (table 1). Thus, even if the proportions of patients categorised as "Monitors" are about the same using the MBSS or TMSI, a large proportion of patients are categorised differently depending on which measure is used.

\section{Relationship between coping styles and psychological distress}

The summarised Monitoring score of the TMSI correlated positively with HAD depression and anxiety as well as with ratings of worry before and after the counselling (table 2). Conversely, the summarised Blunter score correlated negatively with these measures. This is what can be expected according to other studies of information seeking coping styles and psychological distress. ${ }^{18}$ However, there were no significant correlations between coping styles measured by the MBSS and psychological distress (table 2). Patients categorised as Monitors according to the TSMI reported higher HAD anxiety and more worry both before and after the visit compared to patients not categorised as TSMI Monitors. Categorisation of patients as Monitors or non-Monitors according to the MBSS did not show any differences in psychological distress (table 3 ).

To investigate differences in psychological distress between Monitors and non-Monitors over time, only the TMSI categorisation was used since the MBSS appeared to be less sensitive in this context. A main group effect was found in that Monitors scored higher than non-Monitors for "worry" ( $F=8.43$, $\mathrm{p}<0.01$ ) (table 3 ). No differences were found between assessments (time) and there were no interactions. There were no significant differences between groups for HAD anxiety but a decrease over time $(\mathrm{F}=6.94, \mathrm{p}<0.05)$. Also an interaction was detected: Monitors scored higher than non-Monitors before the counselling, but the scores of the Monitors decreased more than that of the non-Monitors $(\mathrm{F}=6.94, \mathrm{p}<0.05)$. For HAD depression, there were no differences between groups but a decrease over time $(\mathrm{F}=4.3, \mathrm{p}<0.05)$. No interactions were detected. A mutation analysis was performed for 25 (40\%) of the participants but only four of these received the result before completing the HAD scale at six months. Thus, 21 participants were waiting for the test results. Of those, Monitors $(\mathrm{n}=9)$ reported more depression $(\mathrm{m}=5.2)$ compared to non-Monitors $(\mathrm{n}=9, \mathrm{~m}=2.4, \mathrm{t}=2, \mathrm{l}, \mathrm{p}<0.05)$. There was no difference for anxiety. This result must be interpreted with caution because of the small number of persons $(n=18$, three did not fill out the questionnaire).

\section{Relationship between coping styles and risk assessments}

Before counselling, $44 \%$ of the participants overestimated their own life time risk for developing cancer, 38\% underestimated it, and only $18 \%$ rated the correct risk. The corresponding figures after the counselling were 25\% overestimated, $18 \%$ underestimated, and $57 \%$ gave a correct estimation.

Table 2 Correlations between coping styles and psychological distress

\begin{tabular}{|c|c|c|c|c|}
\hline \multirow[b]{3}{*}{ Distress $(n=53)$} & \multicolumn{4}{|c|}{ Coping styles } \\
\hline & \multicolumn{2}{|l|}{ MBSS } & \multicolumn{2}{|l|}{ TMSI } \\
\hline & Monitoring & Blunting & Monitoring & Blunting \\
\hline \multicolumn{5}{|l|}{ Worry } \\
\hline Before & 0.19 & 0.07 & $0.39 * *$ & $-0.32 *$ \\
\hline After & 0.18 & 0.01 & $0.35^{* *}$ & $-0.32^{*}$ \\
\hline \multicolumn{5}{|l|}{ HADS anxiety } \\
\hline Before & 0.14 & 0.21 & $0.45^{* *}$ & $-0.31^{*}$ \\
\hline After $†$ & 0.10 & 0.13 & 0.15 & -0.27 \\
\hline \multicolumn{5}{|l|}{ HADS depression } \\
\hline Before & 0.01 & 0.15 & $0.35^{* *}$ & -0.17 \\
\hline After $†$ & -0.12 & 0.19 & 0.26 & -0.24 \\
\hline
\end{tabular}

Table 3 Differences in psychological distress between Monitors and non-Monitors

\begin{tabular}{|c|c|c|c|c|c|c|}
\hline \multirow[b]{2}{*}{$n=53$} & \multicolumn{3}{|c|}{ TSMI Monitors } & \multicolumn{3}{|c|}{ MBSS Monitors } \\
\hline & Yes (mean) & No (mean) & $t$ & Yes (mean) & No (mean) & $t$ \\
\hline \multicolumn{7}{|l|}{ Worry } \\
\hline Before & 3.9 & 2.4 & 3.0 ** & 3.2 & 3.0 & 0.4 \\
\hline After & 3.6 & 2.5 & $2.2^{*}$ & 2.8 & 2.9 & 0.3 \\
\hline \multicolumn{7}{|c|}{ HADS anxiety } \\
\hline Before & 8.1 & 4.7 & 3.0 ** & 6.4 & 5.6 & 0.6 \\
\hline After $†$ & 5.4 & 4.6 & 0.7 & 4.6 & 4.8 & 0.2 \\
\hline \multicolumn{7}{|c|}{ HADS depression } \\
\hline Before & 4.1 & 2.6 & 1.6 & 3.2 & 3.3 & 0.1 \\
\hline Aftert & 3.3 & 2.0 & 1.4 & 2.0 & 3.0 & 1.2 \\
\hline
\end{tabular}


Table 4 Correlations between coping styles and satisfaction with counselling

\begin{tabular}{|c|c|c|c|c|c|c|}
\hline \multirow[b]{2}{*}{$\begin{array}{l}\text { Coping styles } \\
(n=49)\end{array}$} & \multicolumn{6}{|l|}{ Satisfaction } \\
\hline & Instrumental & Affective & Procedural & $\begin{array}{l}\text { Information } \\
\text { (single item) }\end{array}$ & $\begin{array}{l}\text { Expectation } \\
\text { (single item) }\end{array}$ & $\begin{array}{l}\text { Satisfaction } \\
\text { (single item) }\end{array}$ \\
\hline \multicolumn{7}{|l|}{ MBSS } \\
\hline Monitoring & -0.13 & 0.09 & -0.13 & -0.31 * & -0.02 & 0.13 \\
\hline Blunting & -0.11 & 0.13 & -0.01 & -0.09 & 0.24 & $0.28 *$ \\
\hline \multicolumn{7}{|l|}{ TMSI } \\
\hline Monitoring & -0.18 & 0.20 & -0.02 & -0.31 * & 0.09 & 0.08 \\
\hline Blunting & 0.17 & -0.16 & -0.02 & $0.28 *$ & 0.08 & -0.10 \\
\hline
\end{tabular}

\begin{tabular}{|c|c|c|c|c|c|c|}
\hline \multirow[b]{2}{*}{$\begin{array}{l}\text { Distress } \\
(n=57)\end{array}$} & \multicolumn{6}{|l|}{ Satisfaction } \\
\hline & Instrumental & Affective & Procedural & $\begin{array}{l}\text { Information } \\
\text { (single item) }\end{array}$ & $\begin{array}{l}\text { Expectation } \\
\text { (single item) }\end{array}$ & $\begin{array}{l}\text { Satisfaction } \\
\text { (single item) }\end{array}$ \\
\hline \multicolumn{7}{|l|}{ Worry } \\
\hline Before & -0.23 & 0.06 & -0.06 & -0.24 & -0.03 & 0.14 \\
\hline After & $-0.33^{* *}$ & -0.01 & -0.10 & -0.13 & 0.11 & 0.06 \\
\hline \multicolumn{7}{|c|}{ HADS anxiety } \\
\hline Before & -0.19 & $0.29 *$ & 0.19 & -0.18 & 0.07 & 0.10 \\
\hline Aftert & -0.14 & 0.24 & $0.30^{*}$ & 0.04 & -0.01 & 0.04 \\
\hline \multicolumn{7}{|c|}{ HADS depression } \\
\hline Before & -0.16 & 0.20 & 0.04 & 0.02 & 0.22 & 0.10 \\
\hline Aftert & -0.02 & 0.17 & 0.18 & 0.12 & 0.11 & 0.11 \\
\hline
\end{tabular}

One way Anovas were performed separately for data collected before and after counselling, comparing subjects who overestimated, underestimated, or gave a correct estimation with respect to the total monitoring and blunting scores (TMSI and MBSS). There were no significant differences with respect to either the monitoring or blunting scores between groups that differed in their ability to estimate their cancer risk correctly.

\section{Relationship between coping styles and satisfaction with counselling}

Participants were highly satisfied with the genetic counselling. The mean values for the three dimensions ("Instrumental", "Affective", "Procedural") varied between 9.9 and 11.7 (maximum score 12) and for the three single items 3.7-3.9 (maximum 4).

There was a negative correlation between monitoring (both TMSI, $\mathrm{r}=-0.31$ and MBSS, $\mathrm{r}=-0.31$ ) and "Information" (single item). Blunting (TMSI, $r=0.29$ ) was positively correlated with "Overall satisfaction" ( single item) (table 4). Participants categorised as Monitors were less satisfied with "Overall information" (single item) (mean 3.4) compared to those patients not categorised as Monitors (mean 3.8, $\mathrm{t}=3.0$, $\mathrm{p}<0.01)$. This suggests that Monitors were less satisfied with the informative aspects of the counselling compared to non-Monitors but no differences were found for the affective or procedural aspects.

\section{Relationship between satisfaction and psychological distress}

Participants who were less satisfied with the "Instrumental" part of the counselling reported higher levels of worry $(\mathrm{r}=-0.37)$ after the counselling and those who were more satisfied with the "Procedural" aspect reported higher HAD anxiety $(\mathrm{r}=0.30)$. Participants with a higher HAD anxiety before the visit reported higher satisfaction with the "affective" domain of the counselling $(\mathrm{r}=0.32)$, that is, participants who had increased anxiety at the time of the counselling were satisfied with the emotional parts of the counselling (table 5).

\section{DISCUSSION}

One purpose of the present study was to compare two measures of monitoring and blunting. One is an assessment of general coping dispositions, the MBSS, and the other is specifically designed for use in threatening medical situations, the TMSI. There was a positive correlation between MBSS and TMSI monitoring scales but no correlation was found for the blunting scales. The MBSS blunting scale consists of statements concerned mainly with avoidance, but the TMSI also includes "satisfaction" items, for example, "I think things will turn out to be alright". Van Zuren $e t a^{27}$ found no relation between TMSI blunting and avoidance. This suggests that the conceptualisation of blunting differs between these instruments. Further, it indicates higher validity of the monitoring scales since two rather different instruments appear to assess the same concept. Other studies have found the blunting scale to have poorer psychometric characteristics as compared to the monitoring scale. ${ }^{38}$ As expected, a positive correlation was found between monitoring and psychological distress and a negative one for blunting. However, this was only true for the TMSI and not for the MBSS. Several studies have reported relations between a monitoring coping style and psychological distress, especially in situations characterised by uncertainty, for example, when waiting for a test result. In such situations, Monitors report higher distress. ${ }^{1822}$ Since only the TMSI monitoring scale correlated with psychological distress in the present study, the TMSI may be more appropriate for use in a genetic counselling setting. Other studies have reported correlations between a monitoring coping style and psychological distress, using the MBSS. ${ }^{18}{ }^{24}$ A potential explanation of this discrepancy is that the MBSS scenarios may differ in their cultural relevance between the USA and Sweden. 
Before the counselling, Monitors (TSMI) reported more worry and anxiety than non-Monitors. However, anxiety but not worry decreased among Monitors after the counselling. This difference between anxiety and worry may be explained by differences in the timing of assessments. Worry was assessed directly before and after the counselling while anxiety was measured one week before and six months after. Thus, the counselling may have had a long term reassuring effect, especially for Monitors for whom anxiety decreased more than it did for non-Monitors. Monitors may also have obtained additional information during the six month period. Lerman et $a l^{11}$ found that monitoring predicted an increase in general psychological distress over a three month follow up period among women who participated in a randomised trial of breast cancer risk counselling, regardless of the type of counselling received. However, several studies have found that information reduces psychological distress in Monitors. ${ }^{21}{ }^{22}$ Phipps and Zinn ${ }^{18}$ found higher distress in Monitors than in Blunters before undergoing amniocentesis. Monitors also experienced more distress during the period of waiting for the result, but the distress decreased to the same level as for Blunters after the results had been communicated. A similar finding has been reported for women with an increased risk for breast/ovarian cancers. ${ }^{24}$

For depression, there were no differences between the groups. Depression is less situation dependent than anxiety, and may not be influenced directly by the use of a coping style in a specific situation.

The present sample is heterogeneous, perhaps most notably with respect to potential cancer diagnoses. An attempt was made to explore the extent to which the correlations between coping styles and psychological distress were replicable across diagnoses. Thus, such correlations were computed separately for the breast, colorectal, and ovarian cancer samples. The overall pattern of correlations replicated well in all diagnostic groups for the HAD variables. For worry, however, the correlations were somewhat weaker for the breast cancer group than for remaining diagnoses. Owing to the small size of these subgroups no correlations reached statistical significance.

Living in a family with suspected hereditary cancers may be a source of anxiety. Monitors have a tendency to seek out information in threatening situations. Studies have found Monitors to be more demanding as patients, they desire more from the physicians, perhaps as a way to reduce their uncertainty. ${ }^{19}{ }^{39}$ Miller $e t ~ a l^{38}$ found that within a primary care setting, Monitors were more likely to want a test to be performed and to want help and advice on how to deal with stress related to their medical condition. Attendance at genetic counselling is voluntary, and it can be assumed that Monitors attend genetic counselling in a higher proportion than non-Monitors. This assumption cannot be investigated in the present study since the categorisation of patients as Monitors or non-Monitors was done on the basis of whether they scored above or below the median of the sum scores in the present sample. In a study of 77 patients with metastatic cancers attending a medical oncology ward, ${ }^{30}$ using the abbreviated version of the MBSS, the mean MB score (monitoring minus blunting totals) was 1.57. A high $\mathrm{MB}$ score indicates a tendency towards information seeking. In the present study, the total MB score was 1.77. This was significantly higher $(t=2.86, \mathrm{p}<0.01$, one sample $t$ test $)$ than the MB score in the study by Steptoe et al. ${ }^{30}$ This might indicate that people attending genetic counselling are more information seeking than cancer patients attending a medical oncology ward.

The participants' ability to estimate their risk was poor. Only $18 \%$ did this correctly before counselling and $57 \%$ did so afterwards. The proportion of misunderstanding of the risk estimate given by the physician was high $(43 \%)$ in the present study. The difficulties of understanding risk figures and a relative inability correctly to estimate one's own risk have been illustrated in several studies. ${ }^{78}$ In the present study, to be cat- egorised as "correct", some discrepancies were allowed between the participants' ratings and the risk figure that was communicated by the physician. In other studies of estimation of one's own life time risk, the criterion for a "correct" answer has been "within $50 \%$ of the physician's estimate". ${ }^{36}{ }^{40}$ In one of these studies, ${ }^{40} 39 \%$ of women with a family history of breast cancer correctly estimated their own life time risk and $48 \%$ overestimated. Risk perception was independent of genetic counselling (half of the women received special genetic counselling, half ordinary information). Evans et a ${ }^{36}$ found that 130 out of 200 women (65\%) correctly (within $50 \%$ ) estimated their own life time risk after counselling.

Overall, the participants were highly satisfied with the genetic counselling. However, Monitors were less satisfied with "Information" than were non-Monitors. The assumption that satisfaction is not a simple function of factual understanding was confirmed in the present study. Monitors were not only less satisfied with "Information", they also reported more psychological distress compared to non-Monitors. Thus, both psychological distress and satisfaction in connection with the counselling seem to be mediated by the person's coping style. This encourages future studies of whether it is possible to adapt the information given to the person's coping style and to make genetic counselling more efficient for supporting Monitors in their cognitive work of adaptation.

There were no differences between Monitors and nonMonitors in their ability correctly to estimate life time risk for developing cancer after the counselling, that is, both groups were equally distributed over the understand/misunderstand categories of risk estimated by the physician. No relations were found between coping style and ability correctly to estimate life time risk for developing cancer. This finding appears to be in conflict with that of Schwartz et al, ${ }^{25}$ who found that high monitoring scores were associated with high perceived risk for ovarian cancer. In that study, Monitors had a higher perceived risk than low Monitors, regardless of their true risk. Thus, the study of Schwartz et al ${ }^{25}$ does not concern over- or underestimation, which is the main focus of the present study.

In conclusion, coping style is related to psychological distress in genetic counselling. In the long run, counselling may have a reassuring effect, but subjects who are Monitors react with greater distress both directly before and after the counselling. They were also less satisfied with the "Information". This may indicate that the information given is not appropriate for people with a monitoring coping style. Coping style was not related to the ability "correctly" to estimate one's own life time risk of developing cancer. The results highlight the importance of adapting information to the person's coping style in order to reduce unnecessary psychological distress in persons attending genetic counselling.

\section{ACKNOWLEDGEMENTS}

Funding from the Swedish Cancer Society supported this study.

\section{Authors' affiliations}

K Nordin, A Lidén, M Hansson, G Berglund, Department of Public Health and Caring Sciences, Uppsala University, Uppsala Science Park, SE-751 85 Uppsala, Sweden

R Rosenquist, Department of Genetics and Pathology, Rudbeck Laboratory, Uppsala University, SE-751 85 Uppsala, Sweden

Correspondence to: Dr K Nordin, Department of Public Health and Caring Sciences, Uppsala University, Uppsala Science Park, SE-75 185 Uppsala, Sweden; karin.nordin@pubcare.uu.se

\section{REFERENCES}

1 Mulvihill J. Clinical ecogenetics: cancer in families. N Engl J Med 1985;312:1569-70

2 Miki Y, Swensen B, Shattuck-Eidens D, Futreal P, Harshman K, Tautigian S, Liu Q, Cochrane C, Bennett L, Ding W. A strong candidate for the breast and ovarian cancer susceptibility gene BRCA1. Science 1994;266:66-71. 
3 Wooster R, Bignell G, Lancaster J, Swift S, Seal S, Mangion J, Collins N. Identification of the breast cancer susceptibility gene BRCA2. Nature 1995; 378:789-92

4 Claus E, Risch N, Thompson W. Genetic analysis of breast cancer in the cancer and steroid study. Am J Hum Genet 1991:48:232-42.

5 Dunlop M, Farrington S, Carothers A, Wyllie A, Sharp L, Burn J, Liu B, Kinzler K, Vogelstein G. Cancer risk associated with germline DNA mismatch repair gene mutations. Hum Mol Genet 1997:6:105-10.

6 Ley P. Communicating with patients. Improving communication, satisfaction and compliance. London: Croom Helm, 1988.

7 Evans D, Burnell L, Hopwood P, Howell J. Perception of in women with a family history of breast cancer. Br J Cancer 1993;67:612-14.

8 Stadler M, Mulvihill J. Cancer risk assessment and genetic counseling in an academic medical center: consultands' satisfaction, knowledge, and behavior in the first year. J Genet Couns 1998;7:1998.

9 Hopwood P. Psychological issues in cancer genetics: current research and future priorities. Patient Educ Couns 1997;32:19-31.

10 Croyle R, Smith K, Botkin J, Baty B, Nash J. Psychological responses to BRCA1 mutation testing: preliminary findings. Health Psychol 1997; 16:63-72.

11 Lerman C, Schwartz M, Miller S, Daly M, Sands C, Rimer B. A randomized trial of breast cancer risk counseling: interacting effects of counselling, education level and coping style. Health Psycho 1996; 15:75-83

12 Decruyenaere $M$, Evers-Kiebooms G, Boogaerts A, Cassiman J, Cloostermans T, Demyttenaere K, Dom R, Fryns JP, Van den Berghe H. Prediction of psychological functioning one year after the predicitive test for Huntington's disease and impact of the result on reproductive decision making. J Med Genet 1996;33:737-43.

13 Tibben A, Duivenvoorden H, Vegter-van der Vlis M. Presymptomatic DNA testing for Huntington disease: identifying the need for psychological intervention. Am J Med Genet 1993;48:137-44

14 Lazarus R, Folkman S. Stress appraisal and coping. New York: Springer Publishing Company, 1984.

15 Ferrero J, Barreto $M$, Toledo M. Mental adjustment to cancer and quality of life in breast cancer patients: an exploratory study. Psycho-Oncology 1994;3:223-32

16 Nordin K, Glimelius B. Reactions to gastrointestinal cancer - variation in mental adjustment and emotional well-being over time in patients with different prognoses. Psycho-Oncology 1998;7:413-23.

17 Watson M, Greer S, Rowden L, Gorman C, Robertson B. Relationship between emotional control, adjustment to cancer and depression and anxiety in breast cancer patients. Psychol Med 1991;21:51-7.

18 Phipps S, Zinn A. Psychological responses to amniocentesis. II. Effects of coping styles. Am J Med Genet 1986;25:143-8.

19 Miller S. Monitoring and blunting: validation of a questionnaire to assess styles of information seeking under threat. J Pers Soc Psychol 1987; 52:345-53.

20 Suls J, Fletcher B. The relative efficacy of avoidant and nonavoidan coping strategies: a meta-analysis. Health Psychol 1985;4:249-88.

21 Miller S, Managan C. The interacting effects of information and coping style in adapting to gynecologic stress: should the doctors tell all? J Pers Soc Psychol 1983;54:224-36.
22 Watkins L, Weaver L, Odegaard V. Preparation for cardiac catheterization: tailoring the content of instruction to coping style. Heart Lung 1986;15:382-9.

23 Miller S. Monitoring and blunting in the face of threat: implications for adaptation and health. In: Montada L, Filipp S, Lerner M, eds. Life crises and experiences of loss in adulthood. Englewood Cliffs, NJ: Erlbaum, 1991.

24 Tercyak K, Lerman C, Peshkin B, Hughes C, Main D, Isaacs C, Schwartz $M$. Effects of coping style and BRCA1 and BRCA2 test results on anxiety among women participating in genetic counseling and testing for breast and ovarian cancer risk. Health Psychol 2001;20:217-22.

25 Schwartz M, Lerman C, Miller S, Daly M, Masny A. Coping disposition, perceived risk, and psychological distress among women at increased risk for ovarian cancer. Health Psychol 1995;14:232-5

26 van Zuuren F, Muris P. Styles of information seeking under threat: personal and situational aspects of monitoring and blunting. Pers Indiv Diff 1991;12:141-9.

27 van Zuuren F, de Groot K, Mulder N, Muris P. Coping with medical treat: an evaluation of the threatening medical situations inventory (TMSI) Pers Indiv Diff 1996;21:21-31

28 Maguire G, Fairbairn S, Fletcher $C$. Consultation skills of young doctors: benefits of feedback training in interviewing students persist. BM 1986;292:1573-8.

29 Ludwick-Rosenthal R, Neufeld R. Preparation for undergoing an invasive medical procedure: interacting effects of information and coping style. J Consult Clin Psychol 1993;61:156-64

30 Steptoe A, Sutcliffe I, Allen B, Combes C. Satisfaction with communication, medical knowledge, and coping style in patients with metastatic cancer. Soc Sci Med 1991;32:627-32.

31 Steptoe A. An abbreviated version of the Miller Behavioral Style Scale. BrJ Clin Psychol 1989;28:183-4

32 Steptoe A, O'Sullivan J. Monitoring and blunting coping styles in women prior to surgery. Br J Clin Psychol 1986;25:143-4

33 Zigmond A, Snaith R. The Hospital Anxiety and Depression Scale. Acta Psychiatr Scand 1983;67:361-70.

34 Aylard P, Gooding J, McKenna P, Snaith R. A validation study of three anxiety and depression self-assessment scales. J Psychosom Res 1987;31:261-8.

35 Moorey S, Greer S, Watson M, Gorman C, Rowden L, Tunmore R, Robertson B, Bliss J. The factor structure and factor stability of the Hospital Anxiety and Depression Scale in patients with cancer. $\mathrm{Br} J$ Psychiatry 1991;158:255-9

36 Evans D, Blair V, Greenhalg C. The impact of counselling on risk perception in women with a family history of breast cancer. Br J Cancer 1994;70:934-8

37 Shiloh S, Avdor O, Goodman R. Satisfaction with genetic counseling: dimensions and measurement. Am J Med Genet 1990;37:522-9

38 Miller S, Brody D, Summerton J. Styles of coping with threat: implication for health. J Pers Soc Psychol 1988;54:142-8.

39 Leventhal $\mathbf{H}$, Diefenbach $M$, Leventhal E. Illness cognition: using common sense to understand treatment adherence and affect cognition interaction. Cogn Ther Res 1992;16:143-63.

40 Rothemund Y, Paepke S, Flor H. Perception of risk, anxiety and health behaviors in women at risk for breast cancer. Int J Med Genet 2001;8:230-9.

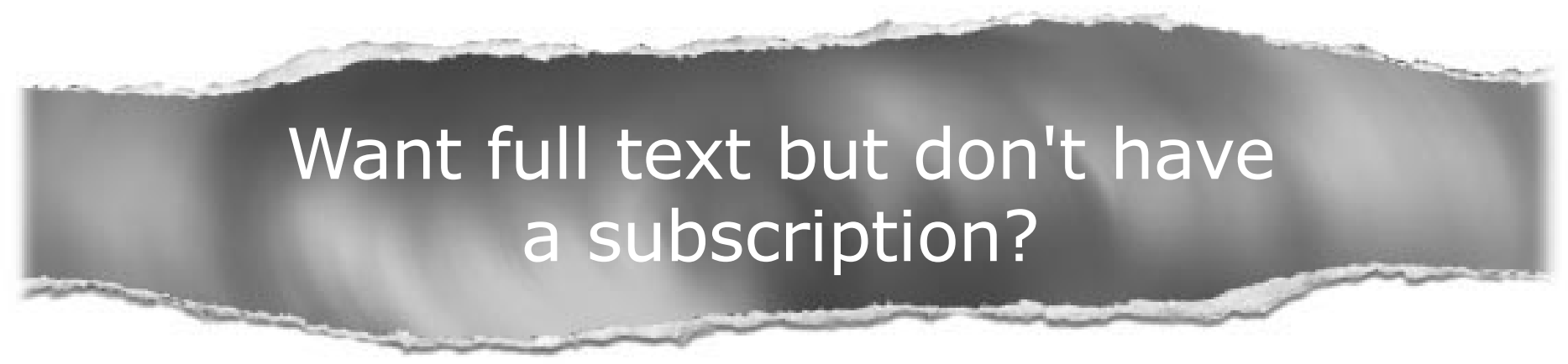

\section{Pay per view}

For just $\$ 8$ you can purchase the full text of individual articles using our secure online ordering service.

You will have access to the full text of the relevant article for 48 hours during which time you may

download and print the pdf file for personal use.

\section{www.jmedgenet.com}

